\title{
Mucosal reactive oxygen metabolite production in duodenal ulcer disease
}

\author{
G R Davies, N J Simmonds, T R J Stevens, A Grandison, D R Blake, D S Rampton
}

\begin{abstract}
To investigate the hypothesis that reactive oxygen metabolites are important in the pathophysiology of duodenal ulcer disease, their production by duodenal mucosal biopsy specimens was measured using luminol and lucigenin amplified chemiluminescence. Luminol chemiluminescence, expressed as background corrected median photon emission $/ \mathrm{mg} / \mathrm{min} \times 10^{3} \quad(95 \% \quad$ confidence intervals), was increased in duodenal inflammation as assessed macroscopically: ulcers $20.3(4.8$ to $51 \cdot 3), n=29$; severe duodenitis 13.9 (6.6 to $75 \cdot 3), n=16$; mild duodenitis $0.0(-0.5$ to 0.8$), \mathrm{n}=56$; controls $-0.8(-1.3$ to -0.1$)$, $n=41 ; p=0.0001$, Kruskal-Wallis) and microscopically: severe $17.0(9.3$ to $51 \cdot 3), \mathrm{n}=12$; moderate $0.3(-2.8$ to 5.8$), \mathrm{n}=17$; mild -0.1 $(-1.8$ to 1.0$), n=17$; controls $-0.8(-1.6$ to $0.0), \quad n=15$; $(p=0.0001)$. Luminol chemiluminescence was directly related to both the macroscopic and microscopic severity of duodenal damage (Spearman's $R=+0.53$, +0.55 respectively, both $\mathrm{p}=0.0001$ ), to histochemical assessment (myeloperoxidase activity) of neutrophil infiltration $(R=+0.63$; $p=0.04$ ), and to lucigenin chemiluminescence $(\mathbf{R}=+\mathbf{0} .56, \quad p=0.0002)$. Luminol chemiluminescence was inhibited by sodium azide $(-80 \%)$, catalase $(-73 \%)$, and dimethyl sulphoxide $(-24 \%)$. Superoxide dismutase inhibited lucigenin more than luminol dependent chemiluminescence $(-61 \%$ and $-7 \%$ respectively, $\mathbf{p}<0.05$ ). Within disease groups, Helicobacter pylori antral infection was associated with increased duodenal chemiluminescence, whereas smoking, alcohol, and use of NSAIDs or $\mathrm{H}_{2}$ blockers had no influence. Their disease related generation in duodenal mucosa supports a role for reactive oxygen metabolites in the pathogenesis of duodenitis and duodenal ulcer. These metabolites might include superoxide, hydrogen peroxide, hydroxyl, and products of myeloperoxidase activity.
\end{abstract}

(Gut 1992; 33: 1467-1472)

Reactive oxygen metabolite (ROM) production by inflammatory cells, while essential for survival, can be inappropriate or excessive, contributing more to host destruction than defence.' Increased ROM production has been linked to the pathogenesis of many inflammatory conditions, including those of the human gastrointestinal tract. ${ }^{2}$ Information directly linking ROM production with duodenal ulceration is mostly confined to animal models. ${ }^{3+}$ One study to date, however, has shown ulcer healing in humans using scavengers or inhibitors of ROM production ${ }^{5}$; in addition, recent data suggest that the pathogenicity of Helicobacter pylori in the upper gastrointestinal tract might be related to stimulation of neutrophil ROM production. ${ }^{6}$

We have now looked for evidence of disease related production of ROMs in duodenal biopsy specimens from patients with duodenal ulcer disease and duodenitis using chemiluminescence assays. We have correlated the results with macroscopic and microscopic appearances and myeloperoxidase activity. To show that ROMs were the oxidants detected by the chemiluminescence assays and in an attempt to identify the type and source of ROM involved, we examined the effects of various enzymes, enzyme inhibitors, and ROM scavengers and compared the results of luminol and lucigenin amplified chemiluminescence responses. We also examined the influence of known risk factors for duodenal ulcer disease such as antral $H$ pylori infection, smoking, alcohol, non-steroidal anti-inflammatory drugs (NSAIDs) and of current use of $\mathrm{H}_{2}$ blockers, on duodenal ROM production.

\section{Methods}

\section{REAGENTS}

Chemicals were obtained from Sigma Chemical Co, Poole, Dorset. Stock solutions of luminol (50 $\mathrm{mg} / \mathrm{ml}$ in dimethyl sulphoxide (DMSO)) and lucigenin $(300 \mu \mathrm{M}$ in phosphate buffered saline (PBS)) were prepared weekly and stored at $4^{\circ} \mathrm{C}$. Other reagents were prepared on the day of each experiment. PBS with added glucose $(5 \mathrm{mM})$, calcium, and magnesium (both $1 \mathrm{mM}$ ) was preoxygenated and adjusted to $\mathrm{pH} 7 \cdot 4$. This solution was used to dilute luminol and lucigenin to a final concentration of $75 \mu \mathrm{M}$, and also as a transport medium for biopsy specimens. Superoxide dismutase-Cu $\mathrm{Zn}$ (SOD) from bovine erythrocytes (final concentration $300 \mathrm{U} / \mathrm{ml}$ ), catalase from bovine liver $(3000 \mathrm{U} / \mathrm{ml})$, and sodium azide $(0.1 \mathrm{mM})$ were made up in PBS. When used as a ROM scavenger, DMSO was used at a final concentration of $5 \%$. Inactivated SOD was prepared by boiling a solution in PBS for two hours, and inactivated catalase by heating the powder to $100^{\circ} \mathrm{C}$ for two hours and then leaving the solution in PBS at room temperature for 24 hours. Inactivation of SOD and catalase was confirmed as previously described. ${ }^{2}$ Reagents for myeloperoxidase staining were obtained from Sigma in kit form.

\section{PATIENTS}

Mucosal biopsy specimens (2-6) were obtained from 145 patients undergoing routine endoscopy at The Royal London Hospital. Patient charac- 
teristics are shown in Table I. These studies were approved by the Tower Hamlets Health Authority Ethical Committee.

TABLE I Patient characteristics

\begin{tabular}{lllll}
\hline & Control & Mild duodenitis & Severe duodenitis & Duodenal ulcer \\
\hline No & 41 & 57 & 17 & 30 \\
Age (range) (yr) & $52(23-83)$ & $49(41-82)$ & $45(26-65)$ & $57(22-80)$ \\
Hpylori+(\%) & 45 & 56 & 64 & 82 \\
Smokers (\%) & 27 & 45 & 71 & 44 \\
NSAID use (\%) & 10 & 18 & 13 & 18 \\
H $_{2}$ antagonist use (\%) & 28 & 46 & 67 & 57 \\
High alcohol use $^{\star}(\%)$ & 3 & 18 & 7 & 8 \\
\hline
\end{tabular}

$\star(>20$ U/week females; $>30 \mathrm{U} /$ week males $)$

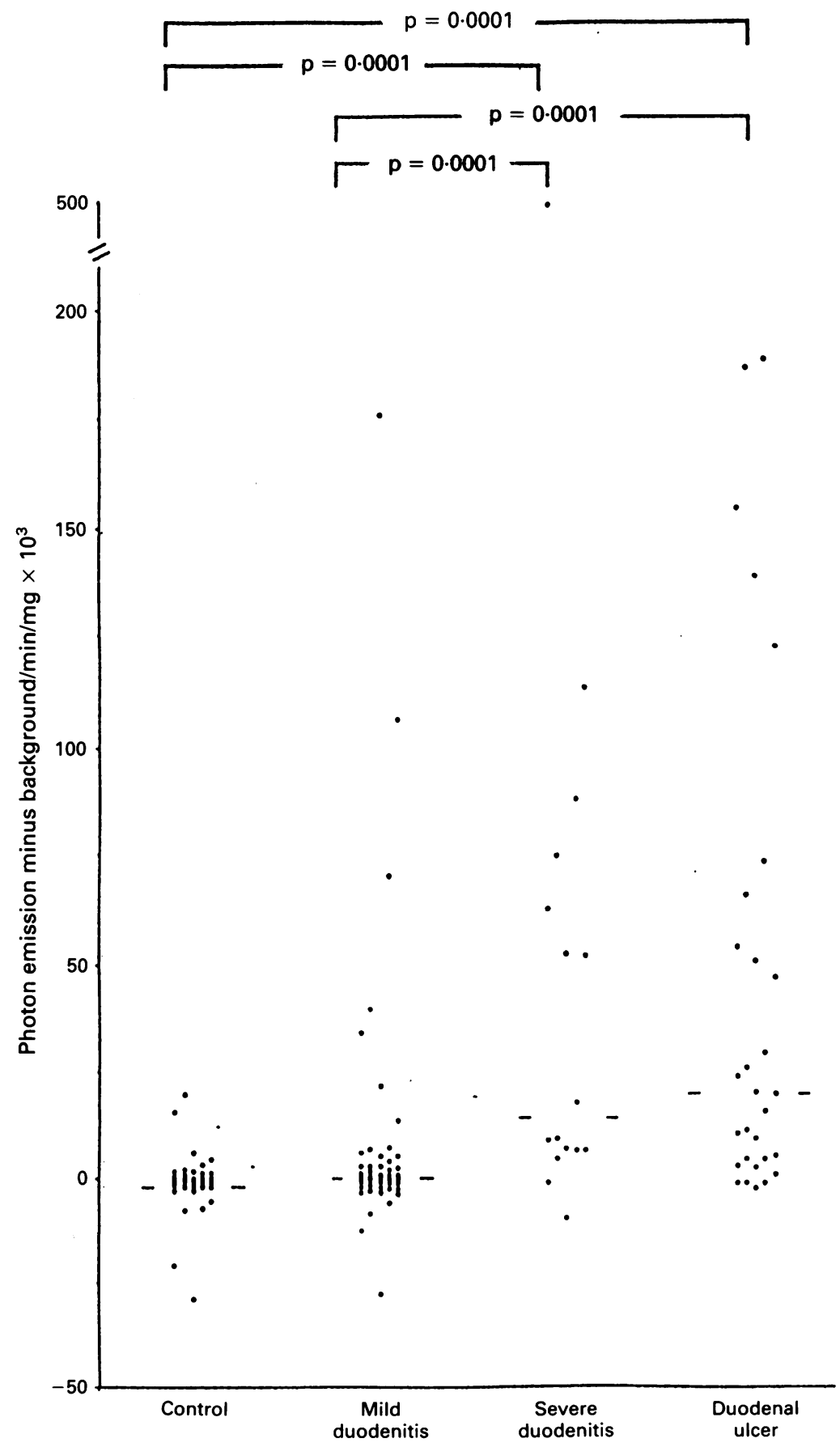

Figure 1: Production of luminol amplified chemiluminescence by duodenal mucosal biopsy specimens according to the endoscopic grade. Results are shown as photons $/ \mathrm{mg} / \mathrm{minute}$ after subtraction of background. Points represent the mean of two or more biopsy specimens from the same patient. Medians and Mann-Whitney $U$ test $p$ values are shown.
TABLE II Histological (haematoxylin and eosin) grading scheme for duodenal biopsies

$\begin{array}{ll}\text { Normal: } & \begin{array}{c}\text { No increase in inflammatory cell infiltrate or } \\ \text { disruption of cellular architecture. } \\ \text { Scanty inflammatory cells with preservation of } \\ \text { glandular and villous architecture. }\end{array} \\ \text { Mild: } & \begin{array}{l}\text { Presence of moderate to severe inflammatory } \\ \text { infiltrate but with preservation of glandular and } \\ \text { villous architecture. }\end{array} \\ \text { Severe: } & \begin{array}{c}\text { Presence of a severe inflammatory infiltrate with one } \\ \text { or more of the following: branching crypts, loss of } \\ \text { villous structure, vascular congestion, ulceration, } \\ \text { glandular disruption. }\end{array}\end{array}$

STUDY PROTOCOL

Drug, alcohol, and cigarette consumption, were recorded. Duodenal inflammation was graded endoscopically according to a modified Lanza scale $^{7}$ as follows: mucosal injection, haemorrhagic and erosive lesions were each scored on a scale of $0-3$, and the three scores summated to give the following groups: control score $=0$, mild duodenitis $=1-3, \quad$ severe duodenitis $=>4$. Duodenal ulcers, considered separately; were defined as a breach in the mucosa greater than $0.5 \mathrm{~cm}$ diameter, with depth. Evidence for $H$ pylori infection was based on results of antral biopsy culture and/or commerical urease (CLO) test. Biopsy specimens (2-6) were taken from the first part of the duodenum for chemiluminescence assay, and, in the case of abnormal mucosa, either from areas of maximum inflammation or the edge of ulceration.

\section{CHEMILUMINESCENCE ASSAY FOR ROMS}

Light emission accompanying the generation of ROMs can be amplified by chemiluminogenic probes such as luminol or lucigenin: these molecules form excited states when exposed to oxidants, relaxing to ground state with the emission of light, detectable using a photomultiplier device. ${ }^{8}$

Mucosal biopsy specimens were washed and transported in preoxygenated PBS; chemiluminescence assays were carried out within three hours using a Packard Tri Carb 1600 CA liquid scintillation analyser operated in the 'out of coincidence' mode. Scintillation tubes containing $1 \mathrm{ml}$ of either $75 \mu \mathrm{M}$ luminol or $75 \mu \mathrm{M}$ lucigenin were precounted for background chemiluminescence. After addition of the biopsy tissue, scintillation tubes were recounted for five minutes. The baseline chemiluminescence for each biopsy is expressed as photons/minute/mg wet weight $\times 10^{3}$ after subtraction of vial background, and the results for each patient were averaged. In paired biopsy specimens from the same site with high chemiluminescence (arbitrarily defined as $>40000$ photons/minute after subtraction of background), inhibitors enzymes, or scavengers affecting ROM production or controls (PBS or heat inactivated catalase or SOD) were added to the vial, and samples were recounted for a further five minutes. Effects of inhibitors and scavengers are expressed as the \% change in chemiluminescence compared with the control value. Immediately after chemiluminescence assays, biopsy specimens were dry blotted, weighed, and preserved for histological analysis (see below). 


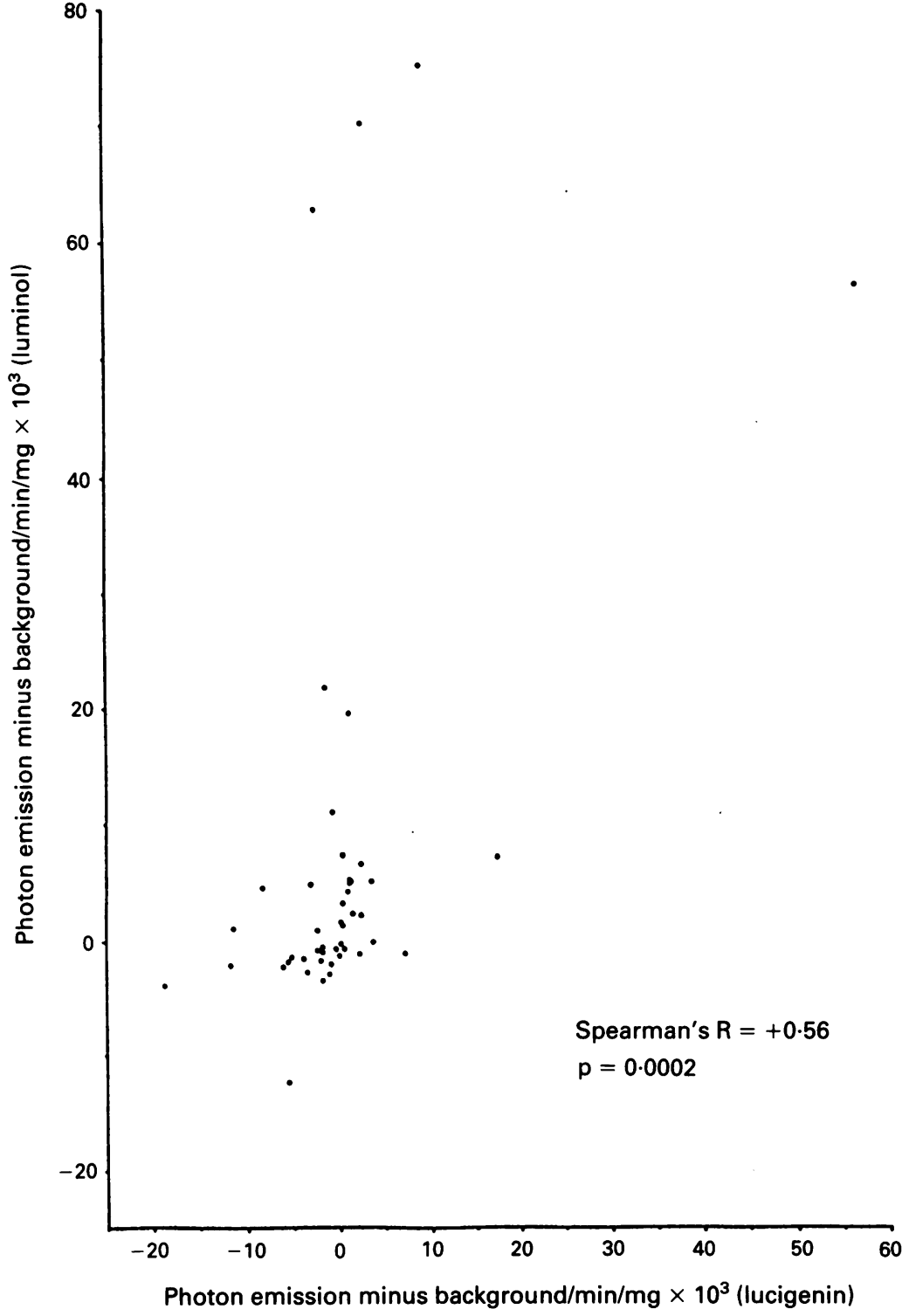

Figure 2: Comparison of chemiluminescence obtained from duodenal biopsy specimens from the same patient using either luminol or lucigenin amplification.

Reproducibility of the method (expressed for each macroscopic group as the median $95 \%$ confidence interval (CI) percentage differences between the second reading and the first, in patients who had two biopsies taken from the same area at the same time) was as follows: controls 22 ( -60 to 66 ) $n=30$ pairs; mild duodenitis 47 ( -38 to 122 ), $n=32$; severe duodenitis 23 ( -128 to 99$), n=24$; ulcers 18 ( -102 to 84$)$, $\mathrm{n}=28$.

\section{MICROSCOPIC ASSESSMENTS}

In 66 patients, haematoxylin and eosin stained sections of formalin preserved biopsy tissues were assessed by two independent pathologists: the semiquantitative grading system used is summarised in Table II. In 11 biopsies, the chemiluminescence result was compared with the myeloperoxidase activity, an index of neutrophil infiltration. ${ }^{9}$ For this assessment biopsies were snap frozen, and later sectioned, fixed for 10 minutes in glutaraldehyde-acetone, and stained according to the Sigma procedure 391
TABLE III Effect of addition of enzymes, inhibitors, and scavengers on reactive oxygen metabolite production by duodenal biopsies. Results are median \% inhibition of chemiluminescence ( $95 \%$ confidence intervals) over five minutes compared with addition of phosphate buffered saline (or inactivated enzyme in the case of superoxide dismutase (SOD) and catalase)

\begin{tabular}{lllll}
\hline & & & \multicolumn{2}{l}{ Chemiluminescence } \\
\cline { 4 - 5 } Agent & Amplifier & No & Inhibition & $(95 \% C I)$ \\
\hline Azide & Luminol & 6 & -80 & $(-77 \text { to }-90)^{\star}$ \\
DMSO & Luminol & 6 & -24 & $(-1$ to -54$)$ \\
Catalase & Luminol & 7 & -73 & $(-61 \text { to }-77)^{\star}$ \\
SOD & Luminol & 8 & -7 & $(-11 \text { to }-56)^{\star}$ \\
SOD & Lucigenin & 5 & -61 & $(-56 \text { to }-90)^{\star} \ddagger$ \\
\hline
\end{tabular}

${ }^{\star} \mathrm{p}<0.05$ compared with control; $\neq \mathrm{p}<0.05$ compared with SOD effects with luminol amplifier; DMSO=dimethyl sulphoxide.

(but using double the stated reaction times), which is based on myeloperoxidase-mediated oxidation of diaminobenzidine. Sections were graded in arbitrary units according to staining intensity. For either method, microscopists were blinded to the other details of the biopsy specimen.

\section{STATISTICS}

Two tailed statistics were used throughout. Chemiluminescence production between the different macroscopic and microscopic groups was compared initially using the Kruskal-Wallis non-parametric test for multiple groups, and subsequent Mann-Whitney $U$ test to look for differences between paired groups. The influence of risk factors within each group was assessed using the Mann-Whitney U test, with corrections for multiple comparisons. Relationships between ROM production and microscopic and macroscopic scores were assessed by Spearman's rank correlation. The effect of inhibitors was assessed using the Wilcoxon signed rank test for paired variables

\section{Results}

\section{CHEMILUMINESCENCE ACCORDING TO}

\section{MACROSCOPIC GRADE}

When duodenal appearances were graded according to the endoscopic appearances, luminol-amplified chemiluminescence (expressed as median photon emission $/ \mathrm{min} / \mathrm{mg}$ wet weight, after subtraction of vial background (95\% CI)) was increased in duodenal ulceration and inflammation: ulcers $20.3 \times 10^{3}(4.8$ to $51 \cdot 3) \mathrm{n}=29$; severe duodenitis $13.9(6.6$ to $75 \cdot 3) n=16$; mild duodenitis $0.0(-0.5$ to 0.8$) \mathrm{n}=56$; controls $-0.8(-1.3$ to -0.1$) n=41 ;(p=0.0001$ KruskalWallis) (Fig 1). Luminol amplified chemiluminescence was positively correlated with the Lanza grading of duodenal mucosa (Spearman's $\mathrm{R}=+0 \cdot 53, \mathrm{p}=0.0001$ ). Luminol and lucigenin chemiluminescence were compared in biopsy specimens from areas of similar macroscopic damage in 41 patients. The results from the two methods were directly related (Spearman's $\mathrm{R}=+0.56, \quad \mathrm{p}=0.0002$ ), however, luminol amplification tended to give greater chemiluminescence (Fig 2). In the smaller number of biopsy specimens assessed for chemi- 


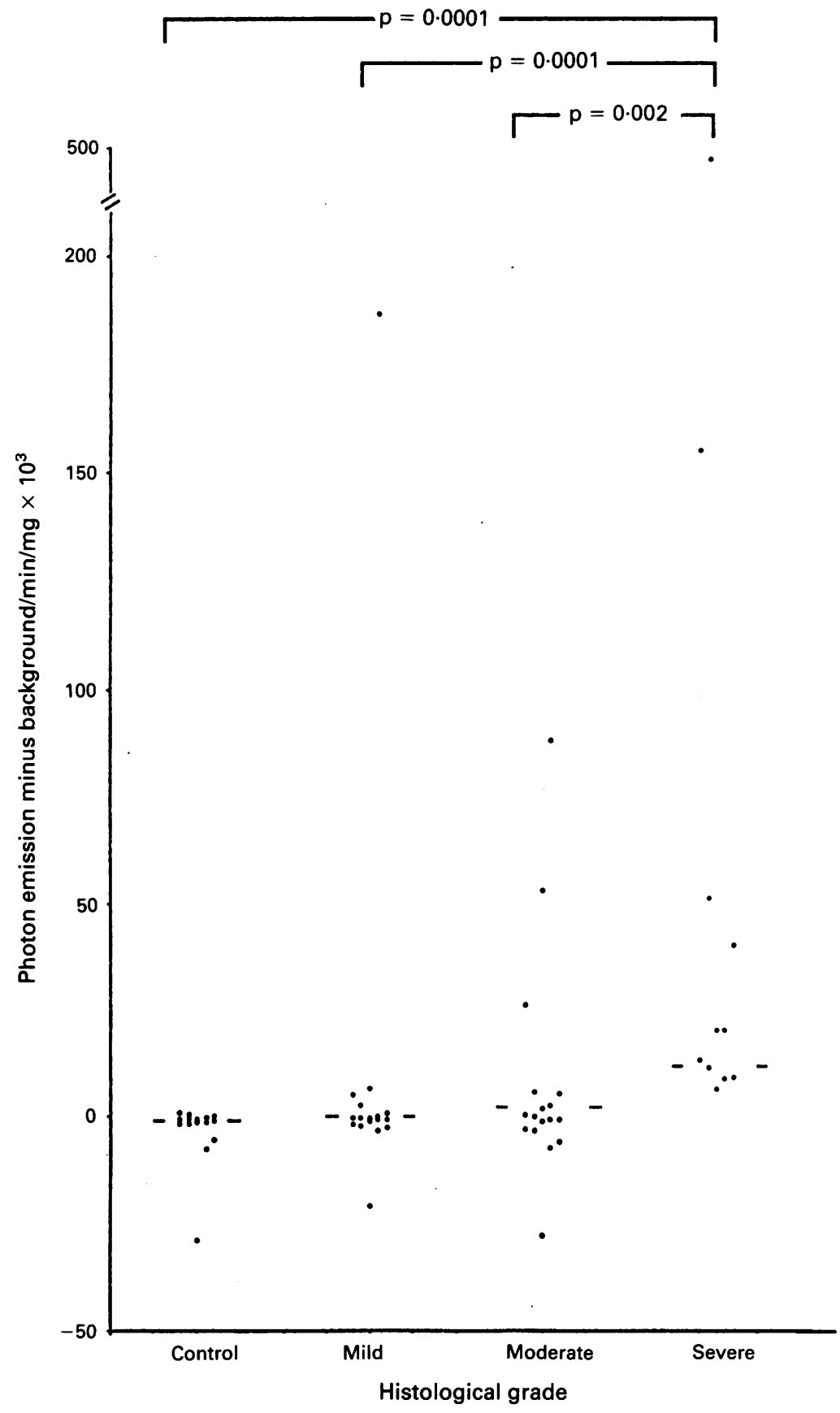

Figure 3: Production of luminol amplified chemiluminescence by duodenal mucosal biopsy specimens according to microscopic (haematoxylin and eosin) assessment of inflammation. Results are shown as photons/mg/minute after subtraction of background. Medians and MannWhitney $U$ test $p$ values are shown.

luminescence with lucigenin, a trend to disease related increases in chemiluminescence did not achieve statistical significance (ulcers and severe duodenitis $0.6(-2 \cdot 3$ to $8 \cdot 1) \mathrm{n}=12$; mild duodenitis $-1.6(-3.5$ to 0.0$) \mathrm{n}=22$; control $-1 \cdot 0(-14 \cdot 0$ to $2 \cdot 1) \mathrm{n}=7 \quad(\mathrm{p}=0 \cdot 2$ KruskalWallis).

\section{CHEMILUMINESCENCE ACCORDING TO} MICROSCOPIC GRADE AND MYELOPEROXIDASE SCORE

As for macroscopic grading, the luminol chemiluminescence result was related to duodenal disease when inflammation was graded microscopically: severe $17 \cdot 0(9 \cdot 3$ to $51 \cdot 3) \mathrm{n}=12$; moderate $0 \cdot 3(-2 \cdot 8$ to $5 \cdot 8) \mathrm{n}=17$; mild $-0 \cdot 1$
$(-1.8$ to $1 \cdot 0) \mathrm{n}=17$; normal $-0.8(-1.6$ to 0.0$)$ $\mathrm{n}=15 ;(\mathrm{p}=0 \cdot 0001$, Kruskal-Wallis) (Spearman's $\mathrm{R}=+0.55, \mathrm{p}=0.0001$ ) (Fig 3). The myeloperoxidase based assay of neutrophil infiltration in duodenal biopsy specimens was also positively correlated with the chemiluminescence result (Spearman's $R=+0 \cdot 63, p=0 \cdot 04, n=11$ ) (Fig 4).

EFFECT OF INHIBITORS (TABLE III)

Luminol amplified chemiluminescence was considerably inhibited by the addition of sodium azide, which inhibits neutrophil myeloperoxidase. There were smaller but significant decreases with catalase (which breaks down hydrogen peroxide) and DMSO (a hydroxyl radical scavenger). SOD (which converts superoxide to hydrogen peroxide and oxygen) produced a greater reduction of lucigenin than luminol amplified chemiluminescence $(\mathrm{p}<0.05)$.

EFFECT OF H PYLORI AND OTHER RISK FACTORS Antral $H$ pylori infection was assessed in 89 patients (Table IV). The increased chemiluminescence in the $H$ pylori positive patients was not simply related to the increased likelihood of more severe duodenal damage than in those who were $H$ pylori negative. Biopsy samples from areas of similar inflammation produced significantly more chemiluminescence if antral $\mathrm{H}$ pylori infection coexisted: conversely, in the absence of duodenal damage antral $H$ pylori status had no effect on the chemiluminescence result.

Luminol amplified chemiluminescence was not significantly different when the whole study group or disease subgroups were subdivided according to presence of the following risk factors for duodenal ulcer disease: smoking, high alcohol consumption $(>30 \mathrm{U} /$ week in men, $>20$ $\mathrm{U} /$ week in women) or use of NSAIDs: similarly, use of $\mathrm{H}_{2}$ blockers had no influence on the chemiluminescence result in any group (data not shown).

\section{Discussion}

ROMs have been implicated in the pathogenesis of human disease characterised by an acute inflammatory cell response, including, in gastroenterology, inflammatory bowel disease, pancreatitis, and hepatitis. ${ }^{10}$ Surprisingly little is known of their role in duodenal ulcer disease. We believe our present results provide the first direct evidence in man for disease related production of ROMs at the site of duodenal ulceration and duodenitis.

There is no natural animal model for duodenal ulcer disease: studies linking ROM production and duodenal injury relate mostly to acute and chronic secretagogue induced experimental duodenal ulceration, where allopurinol, DMSO, and other ROM scavengers have had dose dependent efficacy in reducing mucosal injury. ${ }^{3}$ "In humans, indirect evidence for a role of ROMs in duodenal ulcer disease has been shown in two studies. A randomised study of 220 patients with healed duodenal ulcer showed lower relapse rates in those taking allopurinol 50 


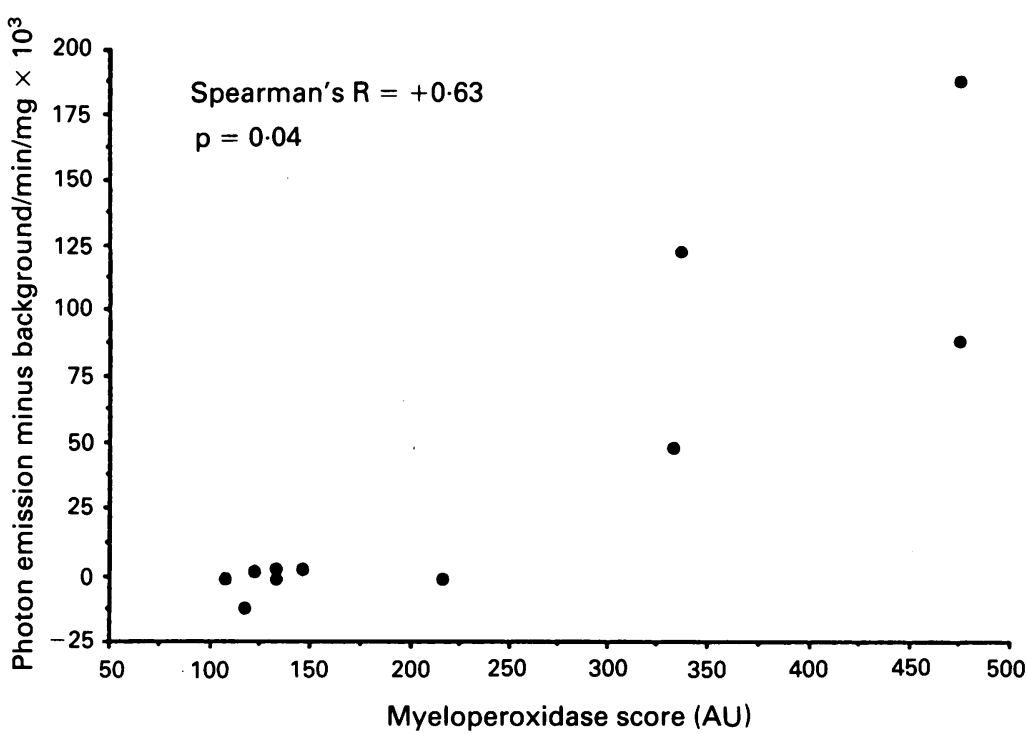

Figure 4: Luminol amplified chemiluminescence according to myeloperoxidase activity. Results are shown as photons/mg/minute after subtraction of background.

$\mathrm{mg}$ four times daily (12\%) or DMSO $500 \mathrm{mg}$ four times daily $(13 \%)$ compared with those taking either placebo (65\%) or cimetidine $400 \mathrm{mg}$ once daily $(30 \%){ }^{5}$ The effects of allopurinol and DMSO were attributed to their ability to scavenge hydroxyl radicals, and in the case of allopurinol, also to inhibition of xanthine oxidase, an enzyme central to ROM production during reperfusion after ischaemia. Jankowski et al found reduced levels of the antioxidant glutathione and increased levels of a marker of ROM mediated damage (malondialdehyde like material) in 23 patients with active duodenal ulcers. In a separate group, glutathione and malondialdehyde like material levels returned towards normal after ulcer healing. ${ }^{12}$ Indirect evidence for increased ROM production has also been found in human gastric ulceration, with reduced amounts of the endogenous antioxidant SOD in biopsy specimens from the edges of active ulcers, and increased amounts in biopsy specimens of healing ulcers. ${ }^{13}$

The chemiluminescence assay we used to demonstrate the presence of mucosal ROMs is sensitive but non-specific. Although any oxidant may act as a substrate for the luminol or lucigenin chemiluminescence reactions, our results support the conclusion that chemiluminescence was produced by the interaction of ROMs with the chemiluminogenic probes. Firstly chemiluminescence correlates strongly with the degree of inflammatory cell infiltrate, whether assessed

TABLE IV Influence of antral Helicobacter pylori infection on chemiluminescence $(C L)$ result, shown as median (photon emission $/$ min $/ \mathrm{mg}$ - background) $\times 10^{3}(95 \%$ confidence intervals), for the study group as a whole, and for the various endoscopic gradings

\begin{tabular}{|c|c|c|c|c|c|}
\hline \multirow[b]{2}{*}{ Population } & \multicolumn{2}{|c|}{$\mathrm{H}$ pylori negative } & \multicolumn{2}{|c|}{$\mathrm{H}$ pylori positive } & \multirow[b]{2}{*}{$p^{\star}$} \\
\hline & No & $C L$ & No & $C L$ & \\
\hline $\begin{array}{l}\text { All biopsies } \\
\text { Control } \\
\text { Mild duodenitis }\end{array}$ & $\begin{array}{l}36 \\
11 \\
17\end{array}$ & $\begin{array}{l}-0.5(-1.6 \text { to } 0.5) \\
-0.4(-1.4 \text { to } 1.4) \\
-0.7(-1.7 \text { to } 0.0)\end{array}$ & $\begin{array}{l}53 \\
10 \\
20\end{array}$ & $\begin{array}{c}4.9(0.2 \text { to } 37 \cdot 6) \\
-0.5(-0.8 \text { to } 1 \cdot 1) \\
1.0(0.1 \text { to } 14 \cdot 6)\end{array}$ & $\begin{array}{l}0 \cdot 0001 \\
0 \cdot 89 \\
0 \cdot 002\end{array}$ \\
\hline $\begin{array}{l}\text { Duodenal ulcer } \\
\text { disease }\end{array}$ & 8 & $3.9(-1.6$ to 30.9$)$ & 24 & $27 \cdot 9(6.0$ to $81 \cdot 2)$ & 0.03 \\
\hline
\end{tabular}

^ Compared with $H$ pylori negative cases in same category by standard histological methods or by (neutrophil specific ${ }^{9}$ ) myeloperoxidase staining. Neutrophil activation, with release of ROMs and myeloperoxidase, is the most likely explanation linking their presence and the chemiluminescence response. Chemiluminescence responses of the same tissue were greater with luminol than lucigenin in almost all cases. This may be explained by presence of peroxidases in the tissue (for example myeloperoxidase from activated neutrophils or lipid peroxidases generated through ROM-mediated damage) as they catalyse the interaction of ROMs with luminol but not lucigenin. ${ }^{+}$

The pattern of response to inhibitors, enzymes, and scavengers specifically affecting ROM production provides the most direct evidence that ROMs were responsible for the chemiluminescence seen, and gives some indication of the particular ROMs present. The most noticeable inhibition of chemiluminescence was found after addition of sodium azide, which is an inhibitor of myeloperoxidase, and thus inhibits both production of hypochlorite $\left(\mathrm{HOCl}^{-}\right)$and the catalysed reaction of superoxide and hydrogen peroxide with luminol. Although sodium azide is a relatively non-specific agent, inhibiting a wide range of cellular processes in addition to neutrophil myeloperoxidase, a direct cytotoxic effect is unlikely at the concentrations used.' SOD produced only a small decrease in chemiluminescence from our biopsy specimens in the luminol system, but larger effects with lucigenin, a pattern repeated when SOD was used to inhibit luminol and lucigenin chemiluminescence from stimulated neutrophils. ${ }^{15}$ The explanation may be that SOD plays a relatively minor part in luminol-amplified chemiluminescence compared with other peroxidase catalysed ROM interactions, particularly with hydrogen peroxide whose levels would increase in the presence of SOD. The moderate inhibitory effects of catalase and DMSO suggest that hydrogen peroxide and the hydroxyl radical also contribute to the chemiluminescence response.

In duodenal ulcer disease, the relationships between chemiluminescence and mucosal damage, and the effects of inhibitors, resemble those we have reported previously in biopsy specimens from patients with inflammatory bowel disease ${ }^{2}$ in the pathogenesis of which a larger body of evidence implicates neutrophil derived ROMs.

The above arguments indicate that the chemiluminescence responses seen in the present study represent duodenal mucosal ROM production The relationship between ROM production and the severity of mucosal injury, taken together with the established pathogenic role of ROMs in experimental models of duodenal ulceration and mucosal damage, and healing of human duodenal mucosal damage with ROM scavengers, strongly, if indirectly, supports a pathogenic role for ROMs in duodenal ulceration and duodenitis.

There are two likely sources of ROMs in the biopsy specimens we studied: (i) activated neutrophils, which produce primarily superoxide and hydrogen peroxide via the cell membrane enzyme NADPH oxidase, and hypochlorite via myeloperoxidase, ${ }^{16}$ and (ii) xanthine 
oxidase, abundant in the upper gastrointestinal mucosa, especially during ischaemia,' which causes generation of superoxide and hydrogen peroxide during subsequent reperfusion. ${ }^{16}$ The two systems are interlinked, as superoxide and other ROM formed during ischaemia/ reperfusion will cause issue damage and increased levels of neutrophil activators and chemoattractants. ${ }^{16}$ Superoxide derived from either neutrophils or xanthine oxidase, spontaneously, and under the influence of SOD, is converted to water and hydrogen peroxide. Hydrogen peroxide is a stable and diffusible radical, but relatively harmless; in the presence of neutrophil derived myeloperoxidase, however, it combines with chloride to form hypochlorous acid, one of the most damaging radicals known. While our results do not exclude the presence in our biopsy specimens of ROMs produced secondary to ischaemia and reperfusion, animal work suggests that neutrophils are the most important source of cytotoxic ROMs in either situation: neutrophil depletion and inhibition of neutrophil adherence effectively prevents intestinal damage after ischaemia and reperfusion. ${ }^{17}$

ROM mediated cytotoxicity is thought to be mainly secondary to lipid peroxidation of cell membranes. ${ }^{18} \mathrm{ROMs}$, including lipid peroxides, may also cause tissue injury by increasing synthesis of cyclo-oxygenase ${ }^{19}$ and lipoxygenase ${ }^{20}$ pathway products of arachidonic acid (results, however, conflicting in different animal models), by stimulating the release of platelet activating factor $^{21}$ and activating the NF-kappaB transcription factor, which causes increased expression of genes coding for a range of proinflammatory mediators, including interleukin 6 and tumour necrosis factor $\alpha .{ }^{22}$

There was no evidence that smoking, alcohol, or use of NSAIDs affected mucosal ROM production within any disease group or in controls. Thus, while promoting duodenal mucosal injury and inflammation, these aetiological factors do not seem to stimulate neutrophils directly. Conversely, duodenal mucosal ROM production was considerably increased in patients positive for $H$ pylori antral infection compared with those with similar macroscopic disease who were $H$ pylori negative. $H$ pylor $i$ have been shown to secrete chemotactic factors ${ }^{23}$ and to stimulate ROM production from human neutrophils in vitro. ${ }^{6}$ Our observations might be explained if $H$ pylori derived chemoattractant and neutrophil stimulating factors had effects distant from the site of infection, or if they promoted neutrophil activation after infection of duodenal ectopic gastric mucosa.

In conclusion, we have shown evidence that duodenal mucosal ROM production is increased in duodenal ulceration and inflammation, particularly in the presence of antral $H$ pylori infection. Our results suggest that antioxidants may contribute to the treatment and prophylaxis of duodenal ulcer disease.

We are grateful for financial support from Searle UK (GRD); the Hilden Charitable Fund (NJS), the National Association for Colitis and Crohn's disease (TRJS), and the Arthritis and Rheumatism Council (DRB).

Some of these data have previously been published in abstract Some of these data have previously been published in abstract
form: Gut 1990; 31: A1183 and Gut 1992; 33 (Suppl 1): S4 and form:

1 Parks DA, Bulkley GB, Granger DN. Role of oxygen-derived free radicals in digestive tract diseases. Surgery 1983; 94: 415-22.

2 Simmonds NJ, Allen RE, Stevens TRJ, vanSomeren RNM, Blake DR, Rampton DS. Chemiluminescence assay of mucosal reactive oxygen metabolites in inflammatory bowel disease. Gastroenterology 1992; 103: 186-96.

3 Salim AS. Role of oxygen derived free radicals in mechanism of acute and chronic duodenal ulceration in the rat. Dig Dis Sci 1989; 35: 73-9.

4 Takamasu M, Fuse Y, Kawamoto K, Ohishi T. Possible mechanisms of diethyldithio-carbamate-induced gastroduodenal mucosal damage in rats. Scand $\mathcal{F}$ Gastroentero Suppl 1989; 162: 112-5.

5 Salim AS. Oxygen-derived free radicals and the prevention of duodenal ulcer relapse A new approach. Am F Med Sci 1990; 300: $1-6$

6 Mooney C, Keenan J, Munster D, Wilson I, Allardyce R, Bagshaw $\mathrm{P}$, et al. Neutrophil activation by Helicobacter pylori. Gut 1991; 32: 853-7.

7 Lanza FL. Endoscopic studies of gastric and duodenal injury after the use of ibuprofen, aspirin and other non-steroid antiinflammiatory agents. Am $\mathcal{F}$ Med 1984; 77: 19-24.

8 Allen RC. Phagocyte oxygenation activities: quantitative analysis based on chemiluminescence. In: Scholmerich J, Andreeson R, Kapp A, Ernst M, Wood WG, eds. Bioluminescence and chemiluminescence. New perspectives. Chichester: John Wiley 1986: 13-22.

9 Krawisz JE, Sharon P, Stenson WF. Quantitative assay for acute intestinal inflammation based on myeloperoxidase activity. Gastroenterology 1984; 87: 1344-50.

10 Cross CE, Halliwell B, Borish ET, Pryor WA, Ames BN, Saul RL, et al. Oxygen radicals and human disease. Ann Intern Med 1987; 107: 526-45.

11 Salim AS. Role of oxygen-derived free radicals in the mechanism of chronic gastric ulceration in the rat: implications for cytoprotection. Digestion 1989; 43: 113-9.

12 Jankowski J, Bridges AB, Scott N, Wormsley KG, Belch J. Circulating free-radical markers and peptic ulcer disease. Eur f Gastroenterol Hepatol 1991; 3: 823-8.

13 Kishi A, Yoshikawa T, Naito Y, Ando T, Yasuda M, Tsujigiwa $M$, et al. Evaluation of superoxide dismutase activity in the gastric mucosa of chronic peptic ulcer patients. Gastroenterology 1990; 98: A 68 .

14 Allen RC. Biochemiexitation: chemiluminescence and the study of biological oxygenation reactions. In: Adam W, Cilento $\mathrm{G}$ eds. Chemical and biological generation of excited states. New York: Academic Press, 1982: 309-44.

15 Allen RC. Phagocytic leucocyte oxygenation activities and chemiluminescence: a kinetic approach to analysis. Meth Enzymol 1986; 133: 449-93.

16 Grisham MB, Granger DN. Neutrophil-mediated mucosal injury. Role of reactive oxygen metabolites. Dig Dis $\mathrm{Sc}$ 1988; 33: 6-15S.

17 Hernandez LA, Grisham MB, Twohig B, Arfors KE, Harlan JM, Granger DN. Role of neutrophils in ischaemiareperfusion-induced

18 Yoshikawa T, Naito Y, Ueda S, Oyamada H, Takemura T, Yoshida $\mathrm{N}$, et al. Role of oxygen-derived free radicals in the pathogenesis of gastric mucosal lesions in rats. $\mathcal{F}$ Clin Gastroenterol 1990; 12 (suppl 1): 565-71.

19 Chakaborti S, Gurtner GH, Michael JR. Oxidant-mediated activation of phospholipase $\mathrm{A} 2$ in pulmonary epithelium. Am $\mathcal{F}$ Physiol 1989; 257: L430-7.

20 Arakawa T, Sakuma H, Kobayashi K. Effects of active oxygen species on prostaglandin and leucotriene synthesis in cultured rat gastric cells. Gastroenterology 1991; 100: A26.

21 Lewis MS, Whatley RE, Cain P, McIntyre TM, Prescott SM, Zimmerman GA. Hydrogen peroxide stimulates the zynthesis of platelet-activation factor by endothelium and synthesis of platelet-activation factor by endothelium and
induces endothelial cell-dependent neutrophil adhesion. $\mathcal{F}$ induces endothelial cell-depender

22 Schreck R, Rieber P, Baeuerle PA. Reactive oxygen intermediates as apparently widely used messengers in the activation of the NF-kappaB transcription factor and HIV-1. EMBOF 1991; 10: 2247-58.

23 Kozol R, McCurdy B, Czanko R. A neutrophil chemotactic factor present in Helicobacter pylori but absent in H Mustelae. Gastroenterology 1991; 100: A101. 